\title{
Spin-flipping with Holmium: Case study of proximity effect in superconductor/ferromagnet/superconductor heterostructures
}

\author{
Daniel Fritsch and James F. Annett \\ H. H. Wills Physics Laboratory, School of Physics, University of Bristol, Bristol BS8 1TL, UK
}

(Dated: July 5, 2021)

\begin{abstract}
Superconductor/ferromagnet/superconductor heterostructures exhibit a so-called long-range proximity effect provided some layers of conical magnet Holmium are included in the respective interface regions. The Ho layers lead to a spin-flip process at the interface generating equal-spin spin-triplet pairing correlations in the ferromagnet. These equal-spin spin-triplet pairing correlations penetrate much further into the heterostructure compared to the spin-singlet and unequal-spin spin-triplet correlations which occur in the absence of Ho. Here we present calculations of this effect based on the spin-dependent microscopic Bogoliubov-de Gennes equations solved within a tight-binding model in the clean limit. The influence of the ferromagnet and conical magnet layer thickness on the induced equal-spin spin-triplet pairing correlations is obtained and compared to available experimental data. It is shown that, in agreement with experiment, a critical minimum thickness of conical magnet layers has to be present in order to observe a sizeable amount of equalspin spin-triplet pairing correlations.
\end{abstract}

PACS numbers: Valid PACS appear here

\section{INTRODUCTION}

The proximity effect arises because superconducting pairing correlations are able to penetrate the interface between a superconductor (SC) and a nonmagnetic metal. The correlations decay in the adjacent metal with a characteristic length scale. Replacing the nonmagnetic metal by a ferromagnet (FM) the pairing correlations become oscillatory and this characteristic length scale is also considerably reduced. The penetration depth of the spinsinglet pairing correlation into the ferromagnetic layer depends on the exchange interaction, which also leads to FFLO-like oscillations 1.2 visible in the ferromagnetic region. The SC/FM interface also generates unequal-spin spin-triplet pairing correlations which have the same oscillating and decaying pattern as for the spin-singlet correlations. However, according to a theoretical prediction by Bergeret et $a l \underline{\underline{3}}$ it should be possible to also create equal-spin spin-triplet pairing correlations, provided the interface allows for some kind of spin-flip process. In principle, these equal-spin spin-triplet pairing correlations should be compatible with a ferromagnetic exchange interaction and allow for much larger penetration depth in the ferromagnetic region of the heterostructure compared to the spin-singlet proximity effect. This socalled long-range proximity effect has stimulated a lot of experimental and theoretical work, and has been summarised in reviews by Buzdin $\underline{4}$ and Bergeret et al.$\underline{5}$

From the experimental point of view several possible sources of spin-flip processes have been identified and have been realised in heterostructure setups. It should be noted that experimental evidence for the long-range proximity effect mostly stems from observations of super currents in SC/FM/SC Josephson junctions with FM length scale incompatible with the spin-singlet proximity effect. Experimentally the heterostructures which have been already realised include half-metallic metals, $\underline{\underline{6} \underline{\underline{8}}}$ introducing a magnetic inhomogeneity at the interface,, noncollinear magnetic interfaces $\stackrel{10-12}{=}$ and helical 13 or conical magnetic structures, $\stackrel{14-16}{\longleftarrow}$ to name but a few.

On the theoretical side, several basically very different approaches have been applied to particular heterostructures to investigate equal-spin spin-triplet pairing correlations and how they are affected by specific spin-flip mediating interfaces. In line with the experimental observations various interfaces which have been studied include half-metallic metals $\stackrel{17.18}{=}$ inhomogeneous magnetisations,$\underline{3,19}$ noncollinear magnetisations,$\underline{20}$ and helical (or conical) magnets $\frac{15,21}{23}$ Green's function techniques based on solutions of the Eilenberger $\frac{17}{17}$ and the Usadel equation have been reported, $, 3,19,20,24,25$ as well as selfconsistent solutions of the Bogoliubov-de Gennes (BdG) equations for suitable tight-binding models $, 21-23,26,27$

Here we present results based on a model heterostructure consisting of a $s$-wave $\mathrm{SC} / \mathrm{FM} / \mathrm{SC}$ junction with additional conical magnet $(\mathrm{CM})$ layers introduced at the interfaces. The relevant tight-binding model is solved in the microscopic spin-dependent BdG equations and solutions are iterated to self-consistency. From the respective eigenfunctions we obtain the different spin-triplet pairing correlations and discuss the influence of CM and FM layer thickness. We find that, in agreement with experimental observations, a critical minimal thickness of CM layers has to be present to observe the long-range proximity effect.

The paper is organised as follows. Sec. Ip provides theoretical background, including the BdG equations and heterostructure setup in Sec. IIA and the spin-triplet pairing correlations in Sec. IIB, respectively. Sec. III is devoted to results, where the influence of FM (CM) thickness on spin-triplet pairing correlations is discussed in detail in Sec. IIIA (Sec. IIIB). A summary and concluding remarks are given in Sec. IV 


\section{THEORETICAL BACKGROUND}

\section{A. Method, heterostructure setup and computational details}

The results presented here are based on self-consistent solutions of the microscopic BdG equations in the clean limit. For the spin-dependent case and incorporating the vector components of a general exchange field $\mathbf{h}$ the BdG equations read $22,28,29$

$$
\left(\begin{array}{cccc}
\mathcal{H}_{0}-h_{z} & -h_{x}+i h_{y} & \Delta_{\uparrow \uparrow} & \Delta_{\uparrow \downarrow} \\
-h_{x}-i h_{y} & \mathcal{H}_{0}+h_{z} & \Delta_{\downarrow \uparrow} & \Delta_{\downarrow \downarrow} \\
\Delta_{\uparrow \uparrow}^{*} & \Delta_{\downarrow \uparrow}^{*} & -\mathcal{H}_{0}+h_{z} & h_{x}+i h_{y} \\
\Delta_{\uparrow \downarrow}^{*} & \Delta_{\downarrow \downarrow}^{*} & h_{x}-i h_{y} & -\mathcal{H}_{0}-h_{z}
\end{array}\right)\left(\begin{array}{c}
u_{n \uparrow} \\
u_{n \downarrow} \\
v_{n \uparrow} \\
v_{n \downarrow}
\end{array}\right)=\varepsilon_{n}\left(\begin{array}{c}
u_{n \uparrow} \\
u_{n \downarrow} \\
v_{n \uparrow} \\
v_{n \downarrow}
\end{array}\right),
$$

with $\varepsilon_{n}$, and $u_{n \sigma}$ and $v_{n \sigma}$ denoting the eigenvalues, and quasiparticle and quasihole amplitudes for spin $\sigma$, respectively. The tight-binding Hamiltonian $\mathcal{H}_{0}$ can be simplified according to 22,30

$$
\mathcal{H}_{0}=-t \sum_{n}\left(c_{n}^{\dagger} c_{n+1}+c_{n+1}^{\dagger} c_{n}\right)+\sum_{n}\left(\varepsilon_{n}-\mu\right) c_{n}^{\dagger} c_{n} .
$$

At multilayer index $n$ we have the electronic creation $\left(c_{n}^{\dagger}\right)$ and destruction operators $\left(c_{n}\right)$, whereas the nextnearest neighbour hopping parameter and the chemical potential (Fermi energy) are chosen to be $t=1$ and $\mu=$ 0 , respectively.

According to Balian and Werthamer ${ }^{31,32}$ the general form of the pairing matrix in Eq. (11) can be rewritten as

$$
\left(\begin{array}{cc}
\Delta_{\uparrow \uparrow} & \Delta_{\uparrow \downarrow} \\
\Delta_{\downarrow \uparrow} & \Delta_{\downarrow \downarrow}
\end{array}\right)=(\Delta+\hat{\sigma} \mathbf{d}) i \hat{\sigma}_{2}=\left(\begin{array}{cc}
-d_{x}+i d_{y} & \Delta+d_{z} \\
-\Delta+d_{z} & d_{x}+i d_{y}
\end{array}\right)
$$

with $\hat{\cdots}$ indicating a $2 \times 2$ matrix, respectively. Making use of the Pauli matrices $\hat{\sigma}$, the superconducting order parameter is described by a singlet (scalar) part $\Delta$ and a triplet (vector) part $\mathbf{d}$, respectively. Defining $\hat{\Delta}$ to contain only the triplet $\mathbf{d}$-vector components yields

$$
\hat{\Delta}=(\hat{\sigma} \mathbf{d}) i \hat{\sigma}_{2}=\left(\begin{array}{cc}
-d_{x}+i d_{y} & d_{z} \\
d_{z} & d_{x}+i d_{y}
\end{array}\right)
$$

Considering this general form of this triplet pairing matrix the product $\hat{\Delta} \hat{\Delta}^{\dagger}$ can be written as

$$
\hat{\Delta} \hat{\Delta}^{\dagger}=|\mathbf{d}|^{2} \hat{\sigma}_{0}+i\left(\mathbf{d} \times \mathbf{d}^{*}\right) \hat{\sigma}
$$

with $|\mathbf{d}|$ describing the gap function and $\mathbf{d} \times \mathbf{d}^{*}$ being a measure for the Cooper pair spin magnetic moment, respectively.

However, here we are interested in the case of $s$-wave $\mathrm{SC}$ only. Therefore the pairing potential is restricted to a scalar quantity $\Delta$ fulfilling the self-consistency condition

$$
\begin{aligned}
\Delta(\mathbf{r})= & \frac{g(\mathbf{r})}{2} \sum_{n}\left(u_{n \uparrow}(\mathbf{r}) v_{n \downarrow}^{*}(\mathbf{r})\left[1-f\left(\varepsilon_{n}\right)\right]\right. \\
& \left.+u_{n \downarrow}(\mathbf{r}) v_{n \uparrow}^{*}(\mathbf{r}) f\left(\varepsilon_{n}\right)\right),
\end{aligned}
$$

where we are summing only over positive eigenvalues $\varepsilon_{n}$ and $f\left(\varepsilon_{n}\right)$ denotes the Fermi distribution function evaluated as a step function for zero temperature. Setting up the multilayer structure as shown in Fig. 1(a) the effective superconducting coupling parameter $g(\mathbf{r})$ equals 1 in the $n_{\mathrm{SC}}=250$ layers of spin-singlet $s$-wave superconductor to the left and right of the heterostructural setup and vanishes elsewhere.

The thickness of the CM layer $n_{\mathrm{CM}}$ is varied from 0 to 25 layers, and we include up to $n_{\mathrm{FM}}=500$ layers of FM in the middle of the heterostructure. The vector components of the conical exchange field are determined by 21,22

$$
\mathbf{h}=h_{0}\left\{\cos \alpha \mathbf{y}+\sin \alpha\left[\sin \left(\frac{\beta y}{a}\right) \mathbf{x}+\cos \left(\frac{\beta y}{a}\right) \mathbf{z}\right]\right\},
$$

with $h_{0}=0.1$ fixing the exchange field in the CM layers, and $a=1$ being the lattice constant. The opening angle $\alpha$ (measured from $+y$ towards $+z$ ) and the turning angle $\beta$ (measured from $+z$ towards $+x$ ) are fixed to the values of Ho, namely $\alpha=80^{\circ}$ and $\beta=30^{\circ}$.

(a)

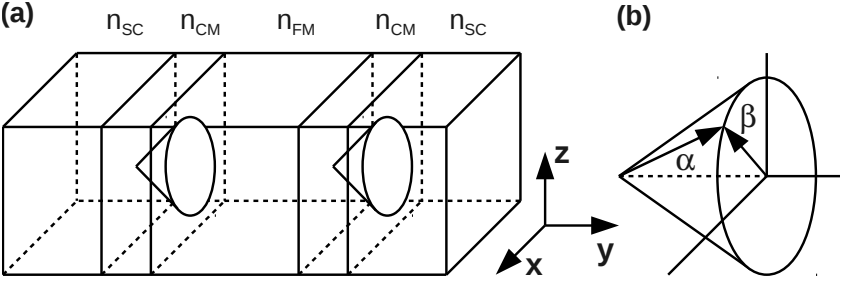

FIG. 1. (a) Heterostructure setup (from left to right): spinsinglet $s$-wave superconductor ( $n_{\mathrm{SC}}$ layers), conical magnet ( $n_{\mathrm{CM}}$ layers), ferromagnetic metal ( $n_{\mathrm{FM}}$ layers), conical magnet $\left(n_{\mathrm{CM}}\right.$ layers), and spin-singlet $s$-wave superconductor ( $n_{\mathrm{SC}}$ layers). (b) Opening and turning angles $\alpha$ and $\beta$ of the conical magnet. According to Eq. (77) $\alpha$ is measured from $+y$ towards $+z$, whereas $\beta$ is measured from $+z$ towards $+x$. 


\section{B. (Triplet) Pairing correlations}

The superconducting pairing correlation between spins $\alpha$ and $\beta$ can generally be evaluated as on-site average for times $t=\tau$ and $t^{\prime}=0$ as

$$
f_{\alpha \beta}(\mathbf{r}, \tau, 0)=\frac{1}{2}\left\langle\hat{\Psi}_{\alpha}(\mathbf{r}, \tau) \hat{\Psi}_{\beta}(\mathbf{r}, 0)\right\rangle,
$$

with $\hat{\Psi}_{\sigma}(\mathbf{r}, \tau)$ being the many-body field operator for spin $\sigma$ at time $\tau$. The time-dependence is introduced through the Heisenberg equation of motion. A pairing correlation evaluated using Eq. (8) is local in space, leading to vanishing triplet contributions for $\tau=0$ in accordance with the Pauli principle ${ }^{26}$ The nonvanishing contributions for finite times $\tau$ are an example of odd-frequency triplet pairing $\underline{\underline{5}}$ Substituting the field operators valid for our setup and phase convention the spin-dependent triplet pairing correlations read

$$
\begin{aligned}
& f_{0}(y, \tau)=\frac{1}{2}\left(f_{\uparrow \downarrow}(y, \tau)+f_{\downarrow \uparrow}(y, \tau)\right)=\frac{1}{2} \sum_{n}\left(u_{n \uparrow}(y) v_{n \downarrow}^{*}(y)+u_{n \downarrow}(y) v_{n \uparrow}^{*}(y)\right) \zeta_{n}(\tau) \\
& f_{1}(y, \tau)=\frac{1}{2}\left(f_{\uparrow \uparrow}(y, \tau)-f_{\downarrow \downarrow}(y, \tau)\right)=\frac{1}{2} \sum_{n}\left(u_{n \uparrow}(y) v_{n \uparrow}^{*}(y)-u_{n \downarrow}(y) v_{n \downarrow}^{*}(y)\right) \zeta_{n}(\tau)
\end{aligned}
$$

depending on position $y$ and time parameter $\tau$ (fixed to $\tau=10$ throughout this work). The $\tau$ dependence is governed via $\zeta_{n}(\tau)$ given by

$$
\zeta_{n}(\tau)=\cos \left(\varepsilon_{n} \tau\right)-i \sin \left(\varepsilon_{n} \tau\right)\left(1-2 f\left(\varepsilon_{n}\right)\right)
$$

The different pairing correlations can be rewritten similarly to the pairing matrix in Eq. (3)

$$
\left(\begin{array}{ll}
f_{\uparrow \uparrow} & f_{\uparrow \downarrow} \\
f_{\downarrow \uparrow} & f_{\downarrow \downarrow}
\end{array}\right)=\left(f_{0}+\hat{\sigma} \mathbf{f}\right) i \hat{\sigma}_{2}=\left(\begin{array}{cc}
-f_{x}+i f_{y} & f_{0}+f_{z} \\
-f_{0}+f_{z} & f_{x}+i f_{y}
\end{array}\right) .
$$

In analogy to Eq. (4) a restriction to the vector components of the $\mathbf{f}$-vector yields the triplet pair function matrix ${ }^{25}$

$$
\hat{f}=(\hat{\sigma} \mathbf{f}) i \hat{\sigma}_{2}=\left(\begin{array}{cc}
-f_{x}+i f_{y} & f_{z} \\
f_{z} & f_{x}+i f_{y}
\end{array}\right) .
$$

Note, that we introduced an additional factor $i$ compared to $\frac{25}{5}$ to be consistent with the definition of $\hat{\Delta}$ in Eq. (4). Instead of Eq. (5) we now have

$$
\hat{f} \hat{f}^{\dagger}=|\mathbf{f}|^{2} \hat{\sigma}_{0}+i\left(\mathbf{f} \times \mathbf{f}^{*}\right) \hat{\sigma}
$$

with the two analogues of $|\mathbf{d}|$ and $\mathbf{d} \times \mathbf{d}^{*}$, being $|\mathbf{f}|$ and $\mathbf{f} \times \mathbf{f}^{*}$, being conveniently expressed in terms of the $\mathbf{f}$ vector components

$$
\begin{aligned}
& f_{x}=\frac{1}{2}\left(-f_{\uparrow \uparrow}+f_{\downarrow \downarrow}\right) \\
& f_{y}=-\frac{i}{2}\left(f_{\uparrow \uparrow}+f_{\downarrow \downarrow}\right) \\
& f_{z}=\frac{1}{2}\left(f_{\uparrow \downarrow}+f_{\downarrow \uparrow}\right) .
\end{aligned}
$$

\section{RESULTS AND DISCUSSION}

\section{A. Influence of ferromagnet thickness $n_{\mathrm{FM}}$}

The self-consistent calculation as described in Sec. II yields the spin-triplet pairing correlations $f_{0}$ and $f_{1}$ as defined in Eq. (9). For an exemplary heterostructure setup with $n_{\mathrm{FM}}=100$ layers and one full turn of the CM to either side, the upper (lower) left panels of Fig. 2 show results on the real (green) and imaginary (orange) parts of the spin-triplet pairing correlations $f_{0}\left(f_{1}\right)$, respectively. The unequal-spin spin-triplet pairing correlation $f_{0}$ clearly shows the expected oscillatory behaviour in the FM region of the heterostructure. These oscillations change in the $\mathrm{CM}$ region and decay in the adjacent $\mathrm{SC}$ layers. In contrast, the equal-spin spin-triplet pairing correlations $f_{1}$ show pronounced features only inside the $\mathrm{CM}$ region and decay into both the $\mathrm{FM}$ and the adjacent SC layers. However, keeping in mind that $f_{1}$ has contributions from $f_{\uparrow \uparrow}$ and $f_{\downarrow \downarrow}$ (see Eq. (9)), the upper (lower) middle panels of Fig. 2 show the real (green) and imaginary (orange) parts of $f_{\uparrow \uparrow}\left(f_{\downarrow \downarrow}\right)$ separately, clearly showing a nonvanishing contribution inside the FM region for the single spin-channels. A more detailed analysis of the influence of CM orientation in the heterostructure on the different spin-triplet pairing correlations can be found in an earlier work ${ }^{22}$ Recalling the pair function matrix as of Eq. (13) the upper and lower right panels of Fig. 2 show the contributions to the magnitudes of the $\mathbf{f}$-vector and $\mathbf{f} \times \mathbf{f}^{*}$, respectively. The magnitude of the $\mathbf{f}$-vector resembles the oscillatory patterns already known from $f_{0}$ (upper left panel). The magnitude of $\mathbf{f} \times \mathbf{f}^{*}$, associated with the spin magnetic moment of the Cooper pairs, shows leakage from the CM layer into the SC layer. However, this leakage shows a strong decay and vanishes after a few layers. On the contrary, there is no such leaking from the CM into the FM region of the heterostructure. 


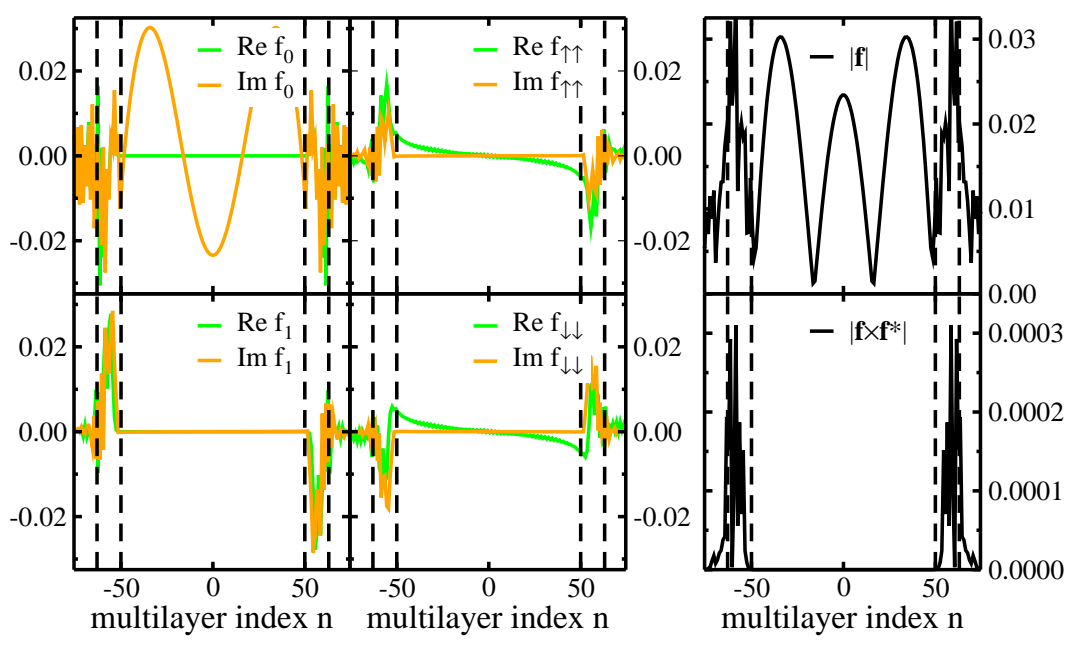

FIG. 2. (colour online) Real (green) and imaginary (orange) parts of the spin-triplet pairing correlations $f_{0}$ (upper left panel) and $f_{1}$ (lower left panel) according to Eq. (9). For better analysis the upper (lower) middle panels depict the real (green) and imaginary (orange) parts of $f_{\uparrow \uparrow}\left(f_{\downarrow \downarrow}\right)$ as components of $f_{1}$ shown in the lower left panel. Upper and lower right panels show the magnitude of the $\mathbf{f}$-vector and the magnitude of $\mathbf{f} \times \mathbf{f}^{*}$ as introduced in Eq. (13), respectively. All data is shown depending on the multilayer index $n$, with $n=0$ lying in the centre of the ferromagnetic layer. The vertical dashed lines indicate the $\mathrm{FM} / \mathrm{CM}$ and $\mathrm{CM} / \mathrm{SC}$ interfaces, respectively.

Keeping the number of CM layers fixed to one full turn we now vary the number of FM layers from $n_{\mathrm{FM}}=0$ to $n_{\mathrm{FM}}=500$ layers. The magnitudes of $f_{0}$ and $f_{1}$ depending on $n_{\mathrm{FM}}$ are depicted in the upper and lower panels of Fig. 3. Similarly to the results presented in Fig. 2 the upper panels in Fig. 3. depicting the magnitudes of $f_{0}$, show a pronounced oscillatory behaviour within the FM region up to $n_{\mathrm{FM}}=500$ layers. There are also much weaker oscillations visible inside the SC region depending on the number of FM layers considered. As expected from the lower left panels of Fig. 2 there is no contribution to the magnitude of $f_{1}$ in the $\mathrm{SC}$ or $\mathrm{FM}$ region of the heterostructure. The only sharp signals originate from the CM regions and are of similar strength as $f_{0}$. The strengths of both, $f_{0}$ and $f_{1}$, are influenced by the number of FM layers $n_{\mathrm{FM}}$, especially visible for small values of $n_{\mathrm{FM}}$, where oscillations are seen in $f_{0}$ and $f_{1}$. Recalling the pair function matrix as of Eq. (13) the magnitudes of the $\mathbf{f}$-vector and $\mathbf{f} \times \mathbf{f}^{*}$ are shown in the upper and lower panels of Fig. 4. respectively. As can be seen from the upper panels the magnitude of the $\mathbf{f}$-vector is quite similar to the magnitude of $f_{0}$ already presented in Fig. 3 Keeping in mind the appearance of the magnitude of $\mathbf{f} \times \mathbf{f}^{*}$ shown in the lower right panel of Fig. 2 there is no influence of the number of FM layers $n_{\mathrm{FM}}$ on this equal-spin pairing correlation. For all $n_{\mathrm{FM}}$ considered here, the strongest contributions to the magnitude of $\mathbf{f} \times \mathbf{f}^{*}$ stem from the CM layers only, with smaller and fast decaying contributions in the adjacent SC layers. No contributions are visible inside the FM layers, as already noted in the discussion of Fig. 2. To summarise this section, the number of FM layers $n_{\mathrm{FM}}$ in the heterostructure has no influence on the magnitudes of $f_{1}$ and $\mathbf{f} \times \mathbf{f}^{*}$, whereas the number of maxima in the magnitudes of $f_{0}$ and the $\mathbf{f}$-vector are increased with increasing number of FM layers $n_{\mathrm{FM}}$.

\section{B. Influence of conical magnet thickness $n_{\mathrm{CM}}$}

Understanding the behaviour of FM layers on the various spin-triplet pairing correlations as discussed in Sec. [IIA we are now interested in the influence of the number of CM layers $n_{\mathrm{CM}}$. For the following calculations we fix the number of FM layers to $n_{\mathrm{FM}}=100$ layers and vary $n_{\mathrm{CM}}$ from 0 to 25 layers, respectively. The magnitudes of $f_{0}$ and $f_{1}$ depending on $n_{\mathrm{CM}}$ are depicted in the upper and lower panels of Fig. 5. Keeping in mind that $n_{\mathrm{FM}}$ is fixed to 100 the upper panels of Fig. 5 show the already familiar oscillatory patterns in the magnitude of $f_{0}$. In contrary to previous results, the magnitude of $f_{0}$ is affected by the number of CM layers $n_{\mathrm{CM}}$, as can be clearly seen in the intensity variations in the FM region shown in the upper right panel of Fig. 5 . As the number of $\mathrm{CM}$ layers $n_{\mathrm{CM}}$ increase, the magnitudes of both, $f_{0}$ and $f_{1}$, show a strongly oscillatory behaviour in the CM region of the heterostructure. Minima appear at multiples of half-integer turns of the full conical magnetic structure, whereas the maxima lie inbetween. Also, there is a phase shift present between the maxima appearing in the magnitudes of $f_{0}$ (upper left panel of Fig. 5) and $f_{1}$ (lower left panel of Fig. 51). In addition, the magnitude of $f_{1}$ show nonvanishing contributions in the FM region of the heterostructure, showing the same oscillatory dependence as in the CM regions, respectively. Apparently, at least a quarter of a full conical magnetic structure has to be present in the heterostructure to obtain a sizable effect on the magnitude of $f_{1}$ (lower panels 

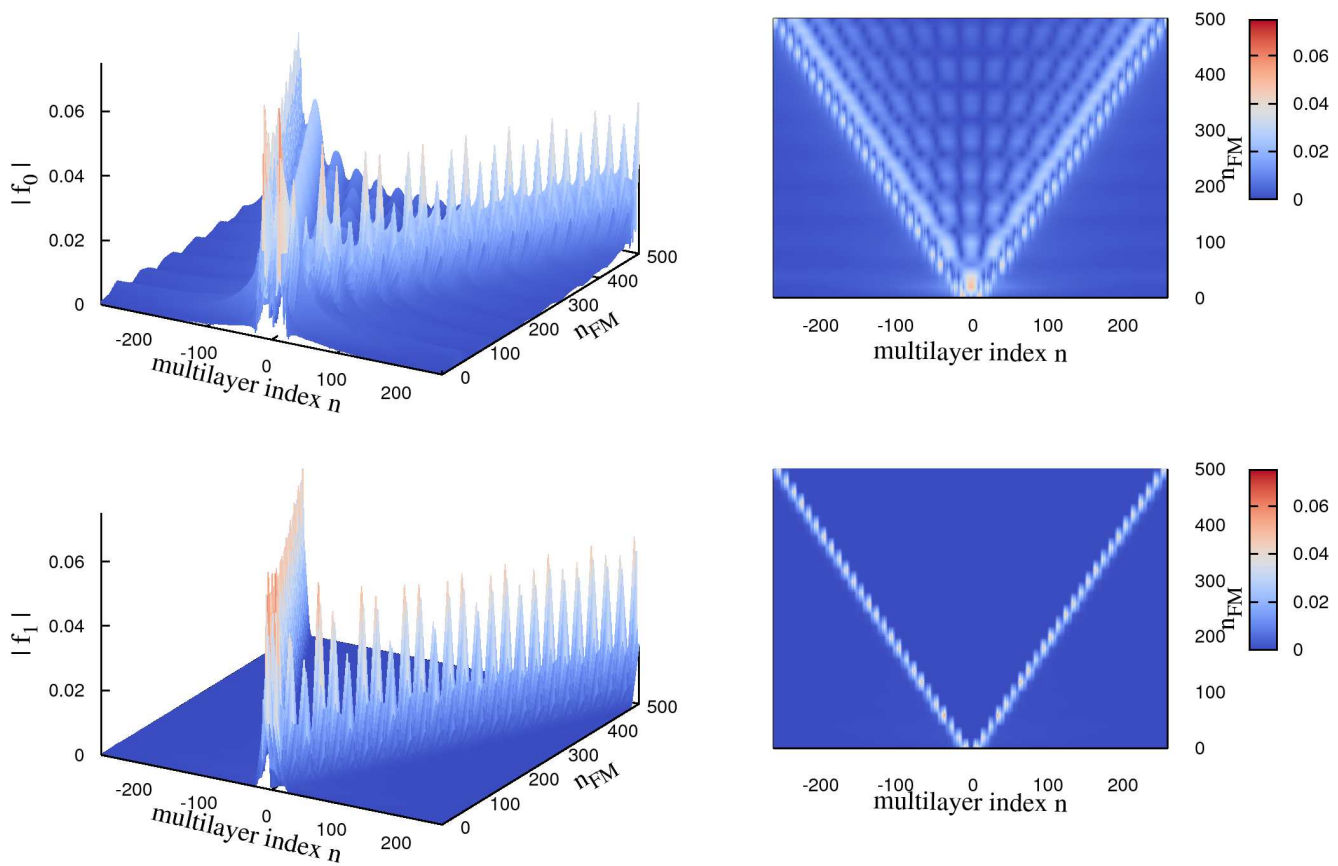

FIG. 3. (colour online) Magnitudes of $f_{0}$ and $f_{1}$ depending on number of FM layers $n_{\mathrm{FM}}$ varied from $n_{\mathrm{FM}}=0$ to $n_{\mathrm{FM}}=500$ layers, respectively. Full data sets are shown in the left panels, whereas the right panels depict a top view of the data.
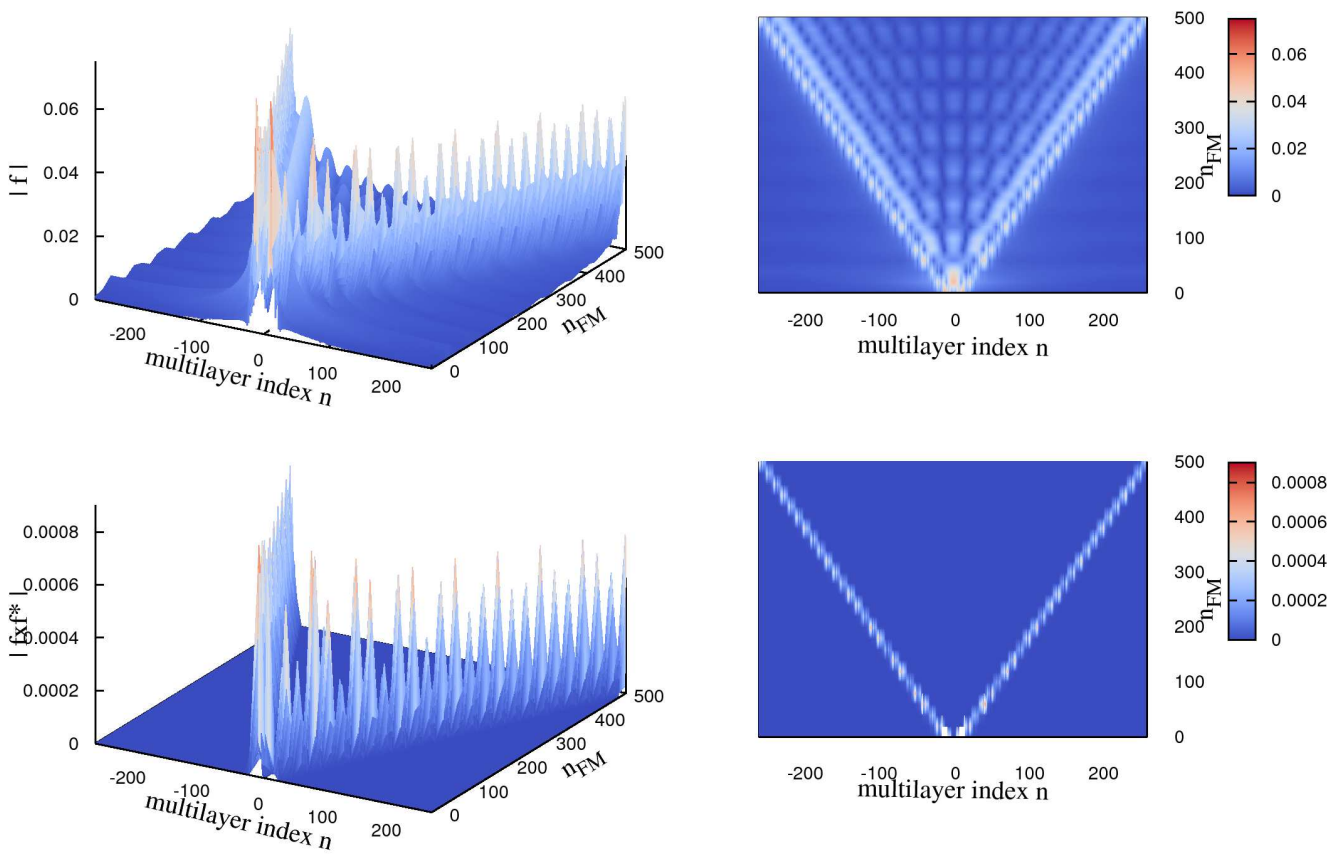

FIG. 4. (colour online) Magnitudes of the $\mathbf{f}$-vector and $\mathbf{f} \times \mathbf{f}^{*}$ as introduced in Eq. (13) depending on the number of FM layers $n_{\mathrm{FM}}$ varied from $n_{\mathrm{FM}}=0$ to $n_{\mathrm{FM}}=500$ layers, respectively. Full data sets are shown in the left panels, whereas the right panels depict a top view of the data.

of Fig. (5). The respective magnitudes of the $\mathbf{f}$-vector and $\mathbf{f} \times \mathbf{f}^{*}$ are shown in the upper and lower panels of Fig. 6] respectively. With the magnitude of $f_{1}$ now showing considerable contributions, the difference between the magnitudes of $f_{0}$ (upper panels of Fig. (5) and the f-vector (upper panels of Fig. 6) is larger compared to Sec. IIIA. The magnitudes of $f_{1}$ are added to those of $f_{0}$ to yield the magnitudes of the $\mathbf{f}$-vector, and are thus responsible for the small intensity variations in the FM region (upper right panel of Fig. 6). With the slight phase shift present 

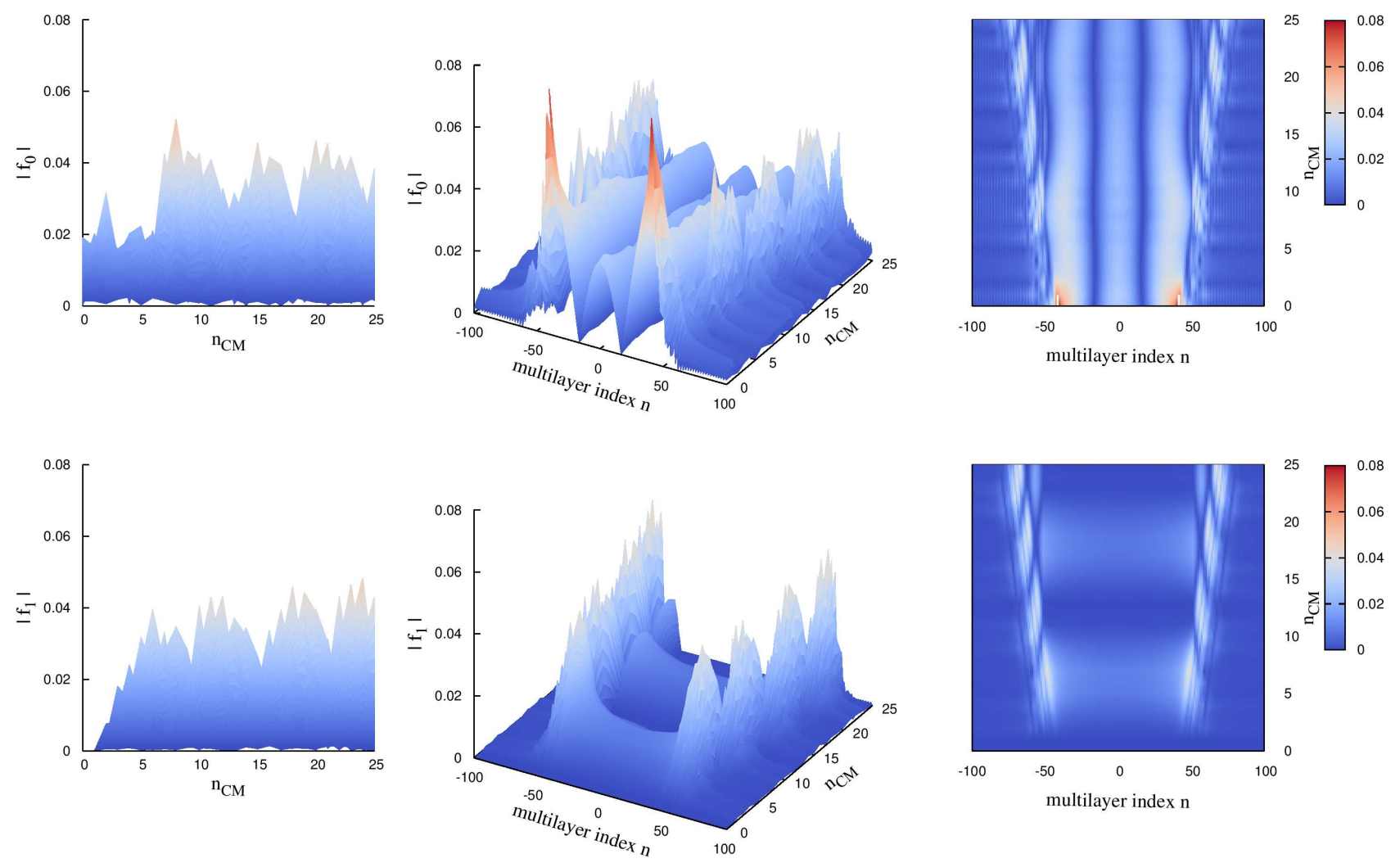

FIG. 5. (colour online) Magnitudes of $f_{0}$ and $f_{1}$ depending on number of CM layers $n_{\mathrm{CM}}$ varied from $n_{\mathrm{CM}}=0$ to $n_{\mathrm{CM}}=25$ layers, respectively. Full data sets are shown in the middle panels, whereas in the left and right panels the same data is shown when viewed from the $y z$ plane (right CM region only) and as a top view, respectively.

between the maxima appearing in the magnitudes of $f_{0}$ and $f_{1}$ the features visible in the magnitude of the $\mathbf{f}$ vector (lower left panel of Fig. 6) are less sharp. From the lower panels of Fig. 6. depicting the magnitudes of $\mathbf{f} \times \mathbf{f}^{*}$, we observe a strong influence of the number of CM layers $n_{\mathrm{CM}}$ on the intensity. This again shows oscillatory behaviour related to the number of turns of the conical magnetic structure. However, in this case there is no increase in the penetration of $\mathbf{f} \times \mathbf{f}^{*}$ into the $\mathrm{FM}$ or SC region. Summarising this section, the number of CM layers $n_{\mathrm{CM}}$ has a more pronounced effect on the spin-triplet pairing correlations compared to the number of FM layers $n_{\mathrm{FM}}$ as discussed in Sec. IIIA The oscillations in the magnitudes of $f_{1}$ shown in the FM and CM regions of the heterostructure are also visible in the magnitude of the $\mathbf{f}$-vector. In addition, the first sizable contributions to the magnitudes of $f_{1}$ and $\mathbf{f} \times \mathbf{f}^{*}$ require at least a number of CM layers corresponding to a quarter of a full conical magnetic turn.

\section{SUMMARY AND OUTLOOK}

In summary, we presented results on the spin-triplet pairing correlations in a $\mathrm{SC} / \mathrm{FM} / \mathrm{SC}$ heterostructure including $\mathrm{Ho}$ as a spin-flip source at the interfaces. The calculations were based on self-consistent solutions of the microscopic BdG equations in the clean limit. In addition to the spin-triplet pairing correlations $f_{0}$ and $f_{1}$, the magnitudes of the $\mathbf{f}$-vector and $\mathbf{f} \times \mathbf{f}^{*}$ have also been calculated. These allow for a deeper understanding of the influence of varying thicknesses of the FM and CM layers on the total and equal-spin spin-triplet correlations, respectively. The leaking of spin magnetic moment of the Cooper pairs into the SC region of the heterostructure is limited and decays rapidly after only a few layers of SC. This behaviour is not influenced by the thickness of FM or CM layers. On the contrary, sizeable contributions to the magnitude of $f_{1}$ require a CM thickness corresponding to at least a quarter of the full magnetic cone structure. This is in agreement with experimental observations $\frac{16}{16}$ where similar heterostructures have been investigated. Thereby, spin-triplet super currents exhibit peak values at $\mathrm{CM}$ thicknesses corresponding to roughly half a turn of the conical magnetic structure.

\section{ACKNOWLEDGEMENT}

This work has been financially supported by the EPSRC (EP/I037598/1) and made use of computational resources of the University of Bristol. 

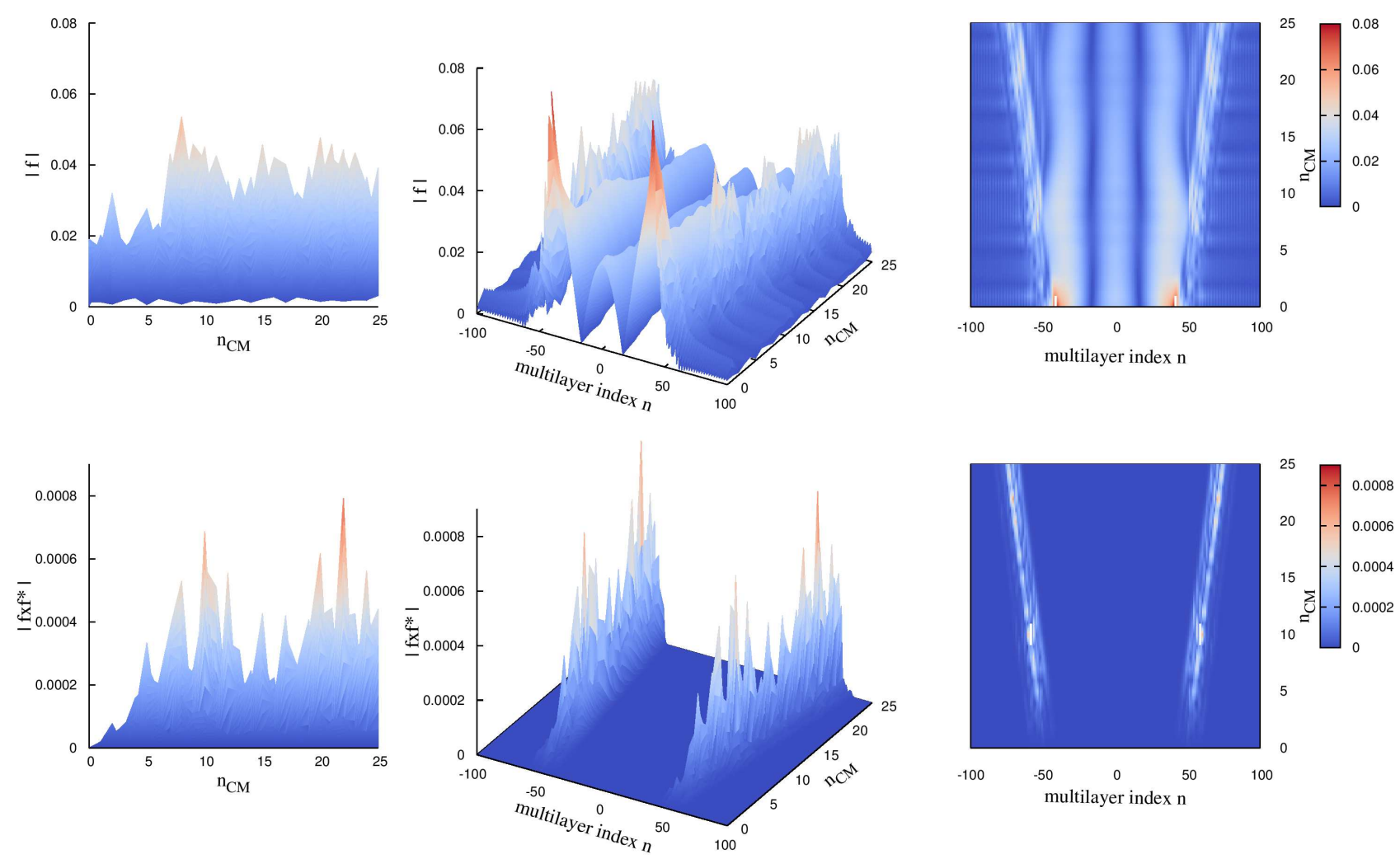

FIG. 6. (colour online) Magnitudes of the $\mathbf{f}$-vector and $\mathbf{f} \times \mathbf{f}^{*}$ as introduced in Eq. (13) depending on the number of CM layers $n_{\mathrm{CM}}$ varied from $n_{\mathrm{CM}}=0$ to $n_{\mathrm{CM}}=25$ layers, respectively. Full data sets are shown in the middle panels, whereas in the left and right panels the same data is shown when viewed from the $y z$ plane (right CM region only) and as a top view, respectively.

1 P. Fulde and R. A. Ferrell, Phys. Rev. 135, A550 (1964).

2 A. I. Larkin and Y. N. Ovchinnikov, Sov. Phys. JETP 20, 762 (1965).

3 F. S. Bergeret, A. F. Volkov, and K. B. Efetov, Phys. Rev. Lett. 86, 4096 (2001).

4 A. I. Buzdin, Rev. Mod. Phys. 77, 935 (2005).

${ }^{5}$ F. S. Bergeret, A. F. Volkov, and K. B. Efetov, Rev. Mod. Phys. 77, 1321 (2005).

${ }^{6}$ R. S. Keizer, S. T. B. Goennenwein, T. M. Klapwijk, G. Miao, G. Xiao, and A. Gupta, Nature 439, 825 (2006).

7 M. S. Anwar, F. Czeschka, M. Hesselberth, M. Porcu, and J. Aarts, Phys. Rev. B 82, 100501 (2010).

8 C. Visani, Z. Sefrioui, J. Tornos, C. Leon, J. Briatico, M. Bibes, A. Barthélémy, J. Santamaría, and J. E. Villegas, Nature Phys. 8, 539 (2012).

9 T. S. Khaire, M. A. Khasawneh, W. P. Pratt Jr., and N. O. Birge, Phys. Rev. Lett. 104, 137002 (2010).

10 C. Klose, T. S. Khaire, Y. Wang, W. P. Pratt Jr., N. O. Birge, B. J. McMorran, T. P. Ginley, J. A. Borchers, B. J. Kirby, B. B. Maranville, and J. Unguris, Phys. Rev. Lett. 108, 127002 (2012).

11 E. C. Gingrich, P. Quarterman, Y. Wang, R. Loloee, W. P. Pratt, Jr., and N. O. Birge, Phys. Rev. B 86, 224506 (2012).

12 V. I. Zdravkov, J. Kehrle, G. Obermeier, D. Lenk, H.A. Krug von Nidda, C. Müller, M. Y. Kupriyanov, A. S.
Sidorenko, S. Horn, R. Tidecks, and L. R. Tagirov, Phys. Rev. B 87, 144507 (2013).

13 G. B. Halász, M. G. Blamire, and J. W. A. Robinson, Phys. Rev. B 84, 024517 (2011).

14 I. Sosnin, H. Cho, V. T. Petrashov, and A. F. Volkov, Phys. Rev. Lett. 96, 157002 (2006).

15 G. B. Halász, J. W. A. Robinson, J. F. Annett, and M. G. Blamire, Phys. Rev. B 79, 224505 (2009).

16 J. W. A. Robinson, J. D. S. Witt, and M. G. Blamire, Science 329, 59 (2010).

17 M. Eschrig, J. Kopu, J. C. Cuevas, and G. Schön, Phys. Rev. Lett. 90, 137003 (2003).

18 M. Eschrig and T. Löfwander, Nature Phys. 4, 138 (2008).

19 M. Alidoust, J. Linder, G. Rashedi, T. Yokoyama, and A. Sudbø, Phys. Rev. B 81, 014512 (2010).

20 A. F. Volkov, F. S. Bergeret, and K. B. Efetov, Phys. Rev. Lett. 90, 117006 (2003).

21 C.-T. Wu, O. T. Valls, and K. Halterman, Phys. Rev. B 86, 184517 (2012).

22 D. Fritsch and J. F. Annett, New J. Phys. 16, 055005 (2014).

23 D. Fritsch and J. F. Annett, J. Phys.: Condens. Matter 26, 274212 (2014).

24 Y. V. Fominov, A. F. Volkov, and K. B. Efetov, Phys. Rev. B 75, 104509 (2007). 
25 S. Kawabata, Y. Asano, Y. Tanaka, and A. A. Golubov, J. Phys. Soc. Jpn. 82, 124702 (2013).

${ }^{26}$ K. Halterman, P. H. Barsic, and O. T. Valls, Phys. Rev. Lett. 99, 127002 (2007).

27 K. Halterman, O. T. Valls, and P. H. Barsic, Phys. Rev. B 77, 174511 (2008).

28 J. F. Annett, Superconductivity, Superfluids and Condensates (Oxford University Press, Oxford, 2004).
29 J. B. Ketterson and S. N. Song, Superconductivity (Cambridge University Press, Cambridge, 1999).

30 L. Covaci and F. Marsiglio, Phys. Rev. B 73, 014503 (2006).

31 R. Balian and N. R. Werthamer, Phys. Rev. 131, 1553 (1963).

32 M. Sigrist and K. Ueda, Rev. Mod. Phys. 63, 239 (1991). 